\title{
PARA UNA CRÍTICA DE LA ESCOLARIZACIÓN DE LOS CUERPOS. EL EJEMPLO ITALIANO
}

Criticism about the schooling of bodies. The Italian example

Por uma crítica a escolarização dos corpos. O exemplo italiano

(1) Donato Antonio

(2) Mancini Pamela

(1) Università di Padova; Associazione Leib, Italia. Correo electrónico: antonio.donato@pdh.unipd.it; infoleib@inventati.org

(2) Associazione Leib, Italia. Correo electrónico: pamelae@libero.it

\section{Resumen}

El proceso moderno de escolarización que persigue el plan económico y político para establecer un cuerpo dócil, útil y productivo, funcional a la reproducción de la cultura burguesa y al sistema económico capitalista, es ahora más que nunca en el centro de muchas críticas. Las formas crecientes de normalización educativa, que en el ejemplo particular italiano excluyen o medicalizan las resistencias encarnadas, están produciendo, de hecho, reflexiones particulares y una politización que a partir del bios y de los cuerpos van a establecer nuevos procesos educativos fuera del sistema del welfare, hacia una biopolítica afirmativa.

Palabras clave: desescolarización, cuerpos, biopolitica.

\begin{abstract}
Modern process of schooling, that pursues the achievement of economic and politic project to build a docile, useful and productive body, functional to reproduce bourgeois culture and capitalist economic system, is nowadays object of critical Growing form of education standardization, that in Italy excluding or medicalize embodied resistors, is doing a reflection and politicization from bios and bodies. Reflection and politicization from bios and bodies are founding new education process, out from welfare system, for an affermative biopolitics.
\end{abstract}

Keywords: deschooling, body, biopolitics. 


\section{Resumo}

O processo moderno de escolarização, que persegue o projeto econômico e político de construção de um corpo dócil e produtivo, funcional à reprodução da cultura burguês e ao sistema econômico capitalista, é hoje mais do que nunca alvo de críticas. As crescentes formas de padronização educativa que, especificamente no exemplo italiano, excluem e medicalizam as resistências encarnadas, estão de fato produzindo uma especifica reflexão e politização que a partir do bios e dos corpos, institui novos processos educativos fora do sistema de welfare, em direção de uma biopolítica afirmativa.

Palavras-chave: desescolarização, corpo, biopolítica.

\section{Intoducción}

«No hay nada que sea más injusto cómo hacer las partes iguales entre desiguales» (Don Milani en Lettera a una professoressa)

En la declinación del Welfare State, la biopolítica está tomando un giro que muchos autores identifican como parte de la bioeconomía (Rose, 2007), en el sentido de uno estructural y directo gobierno del económico sobre las dinámicas del viviente. Robert Castel (2004) también explica la gran capacidad del sistema del welfare en crisis, de estructurar las subjetividades poniéndolas orgánicamente en un modelo de regulación disciplinar, un dispositivo que identifica los sujetos y que da acceso a los derechos y garantías sociales, con efectos de integración, sino también de exclusión y marginación.

El seguiente artículo, sobre la base de estas reflexiones y a través del ejemplo de la escuela italiana, trata de describir cómo la escuela, insertada en el sistema del welfare, puede ser entendida como un dispositivo disciplinar, que tiene como objetivo la producción de subjetividades con el resultado de una paradójica sujeción (Foucault, 1975a). Específicamente, el texto se enfocará en las técnicas de medicalización ejercidas sobre los cuerpos, que se definirán a través de la metáfora del lecho de Procusto. Estas técnicas, gracias al desarrollo de disciplinas psy, constituyen nuevos métodos de gobierno en las prácticas de sujeción en campo de la educación, formas de 
autoridad política para la normalización del sujeto desviado y de su re-inclusión en el mismo sistema, sino también de su marginación para neutralizar las críticas encarnadas.

También por un empobrecimiento del análisis de los procesos educativos, ya no apoyada por la sociología o la antropología, la perspectiva neuropsiquiátrica llega a ocupar un lugar central en esos estudios, ofreciendo una lectura epistemológica con base orgánica a la desviación individual y social, definiendo normal y anormal el sujeto a partir de su cerebro, y al mismo tiempo define la normalidad como un estado natural que puede ser restaurado a través de la intervención técnica y farmacológica. La perspectiva hegemónica bio-psico-social, que también orienta las intervenciones educativas, de hecho, si por un lado revela una tímida apertura a la vida cultural, política y social de la subjetividad, por el otro establece el orden de las jerarquías disciplinarias destinadas a producir y gobernar el sujeto desviado.

Como dicen los estudios post-foucaultianos de los Disability Studies (Abberley, 1987; Oliver, 1990; Barton, 2001) y de los Governmentality Studies (Barry, Osborne, Rose, 1996; Burchell, Gordon, Miller, 1991), el aumento exponencial de las certificaciones de discapacidad en las escuelas italianas (Istituto Nazionale di Statistica [ISTAT], 2015), es de entenderse principalmente como una crisis más amplia de la gobernabilidad, governance que encuentra en el mecanismo de medicalización una estrategia de poder para someter y paralelamente objetivar el desviado.

El objetivo de este trabajo es mostrar cómo la crítica y la politización de esos efectos, induce grupos cada vez más grandes y heterogéneos entre ellos para luchar por la "reapropiación" de los procesos educativos en espacios sociales que no pertenecen ni al mercado económico ni al sistema de welfare. Estas fuerzas definibles "instituyentes" por su impulso innovador y transformador -por ejemplo la red nacional Tutta un'altra scuola-, no sólo asumen las críticas pasadas elaboradas por la pedagogía activa (Bini, 1971), o no autoritaria (Facchinelli, Vaiani, y Sartori, 1971), por la necesidad de una renovación metodológica y didáctica de los procesos educativos, sino que intentan llevar a cabo una reversión de los efectos del poder "sobre" la vida hacia una biopolítica “de la" vida de un bios politizado (Esposito, 2004), dónde está central la política "de los" cuerpos, que a través de prácticas educativas innovadoras permiten acceso a nuevos procesos de subjetivación. 


\section{La disciplina del cuerpo: escuela como estrategia biopolítica}

La palabra disciplina es seguramente una de las expresiones más ambivalente: puede significar educación y aprendizaje, pero quiere decir también punición, castigo, autoridad, y represión; puede ser un curso específico de enseñanza, pero también una competición como en el caso de las disciplinas deportivas.

De hecho, si profundizamos su análisis desde las genealogías foucaultianas sobre las transformaciones sociales del siglo XVIII de la sociedad occidental, por disciplina podemos entender de manera ambivalente tanto un descubrimiento del cuerpo como objeto y el objetivo de poder, cuanto la génesis del conocimiento sobre el cuerpo, en la que el "poder de castigar", "no es esencialmente diferente de la de sanar o educar" (Foucault, 1975b). Sobre la base de los nuevos principios de la burguesía de "gentilezaproducción-lucro", el cuerpo es atravesado por un trabajo "meticuloso" y "macizo" para individualizar y normalizar actitudes y comportamientos. Como nos recuerda el mismo Foucault, "el poder lejos de impedir el conocimiento, lo produce" y "si fue posible establecer un conocimiento sobre el cuerpo, ha sido a través de un conjunto de disciplinas militares y escolares. Es solamente a partir de un poder sobre el cuerpo que un saber fisiológico, orgánico fue posible" (1975b:4, nuestra traducción). El poder disciplinario que sustituye el antiguo poder soberano no sólo fuerza, sino que conduce, controla, regula una multiplicidad de acciones y obra estructurando el campo de posibles acciones. De hecho, si hoy podemos hablar de biopolítica, es exactamente la toma de la vida por el poder, más que la amenaza de matar, que le da acceso al cuerpo. El poder-saber pedagógico es, pues, un dispositivo de disciplina "que ejerce un efecto positivo sobre la vida, que comienza a manejarla, a fortalecerla, a multiplicarla, a ejercer sobre ella los controles precisos y ajustes generales" (Foucault, 1976:179-180, nuestra traducción). Estos controles no sólo se llevan a cabo a través de la consciencia, sino especialmente en y con el cuerpo, que es una realidad biopolítica en la que la escuela como la biomedicina toma la forma de una estrategia de gobierno. Los programas escolares son, de hecho, algo más que la mera descripción de los contenidos, son campos de lucha y de conflicto, para tratar de imponer la hegemonía cultural, son las teorías espaciales de la cognición y del desarrollo del cuerpo y, como tales, contienen visiones del mundo. La idea implícita de control social dentro del plan de estudios se indica por la pedagogía crítica como el "programa oculto". Este plan de 
estudios se puede considerar como el conjunto de conocimientos, valores $\mathrm{y}$ comportamientos que no se detallan en el plan de estudios oficial, pero se aprenden de manera implícita por las relaciones sociales establecidas, de los rituales, de las prácticas y de las configuraciones espaciales y temporales de la escuela, como se verá incorporadas.

En este sentido tenemos que, por lo tanto, decir con Esposito que "no hay que una política de los cuerpos, en los cuerpos y a través de los cuerpos" (Esposito, 2004:86, nuestra traducción) y que "la educación en cualquiera de sus aspectos, se compone principalmente de un sistema de técnicas que basan su eficacia en la ejecución de su materialidad a un sistema de cuerpos" (Massa, 1986:367, nuestra traducción). El error, por lo tanto, es pensar que las prácticas del cuerpo dentro de la escuela son solamente las de educación física, ignorando el enorme trabajo que se realiza en el aula en las otras disciplinas para hacer un cuerpo escolarizado, o "dócil”. La educación física -obligatoria en Italia ya con la ley estatal desde mediados del siglo $\mathrm{XIX}^{1}$ - aunque no representa la totalidad de las prácticas del cuerpo en la escuela, subraya a través su genealogía las estrategias biopolíticas que controlan y regulan el cuerpo. Si durante mucho tiempo se consideró una estrategia adecuada para la dominación burguesa que pretende construir un trabajador dócil políticamente y con rédito económico, en lo que el sufijo "racional" estaba en línea con el pensamiento taylorista de la "teoría racional del trabajo", con el proceso de "sportivisation" la educación física se trasformó en un dispositivo que puede proporcionar un habitus en línea con los principios de competición y de meritocracia, que pone el énfasis en el esfuerzo individual. Esto ya antes de las pedagogías emergentes de las competencias. Hoy, en Italia, la educación física con nuevos paradigmas que se caracterizan por un nuevo control social distinguido por la relación entre la governance y la neurociencias que la transforman en la ciencia motoria, redefine la subjetividad en términos psicológicos y neuroquímicos, desempeñando esencialmente el papel de fármaco. Esa relación permite de dejar caer cada fracaso escolar, no en el plano cultural, es decir, en las decisiones políticas y socioeconómicas de un mercado neoliberal, sino sólo en un sí reducido a "neuroquímica". La peor de las ilusiones, dijo Renè Lourau (1969) es creer que el

${ }^{1}$ Referencia a la ley del Estado italiano (n. 4442, 7 luglio 1878 art.1). 
conocimiento pedagógico se puede separar de las condiciones políticas y sociales de su transmisión. Por eso vamos a tratar, de acuerdo con la obra de Bourdieu y Passeron, de pensar la escuela como un dispositivo de reproducción social que adopta la cultura particular de las clases dominantes, enmascara la naturaleza social y la presenta como la naturaleza objetiva, sin lugar a dudas, negando al mismo tiempo las culturas de otros grupos o clases sociales (Bourdieu y Passeron, 1970). El capital reproducido de la escuela, siguiendo a Bourdieu, puede ser llamado con el nombre de "capital cultural", que se divide en capital cultural "objetivado" como las calificaciones educativas, y un capital cultural "incorporado", que es un patrimonio cultural hecho no sólo de conocimiento conceptual, sino también del conocimiento incorporado. Con el fin de analizar el último, es fundamental combinar una perspectiva fenomenológica, atenta a la subjetividad y a la experiencia de los actores sociales, con un enfoque económicopolítico, capaz de captar la acción de fuerzas macro-sociales más amplias. Un análisis es posible a través del paradigma de incorporación que se desarrolla a partir de dos conceptos: uno está dado por la filosofía fenomenológica de Marleau-Ponty que pone el cuerpo como "estar en el mundo", por el cual el cuerpo sería no tanto un objeto para ser estudiado en relación con la cultura, sino que se debe considerar como sujeto de la cultura; el otro es el concepto bourdiano de "habitus", entendido como un verdadero capital cultural que se produce como incorporación, es decir, como una experiencia corporal producida por las condiciones de socialización del individuo, y se produce bajo la apariencia de lo que parece ser absolutamente innato. En este sentido, es interesante mencionar el estudio de Prout (1989), que mostró cómo niños y niñas en las escuelas primarias, a través de su intensa participación en deportes de contacto físico como el fútbol, respondieron de manera diferente a las enfermedades de sus compañeros: las niñas incrementaban atención e interés (expresadas a través de visitas a los hogares y los buenos deseos para una pronta recuperación), mientras los niños consideraban la debilidad de la enfermedad como algo con lo cual competir. Este ejemplo subraya cómo la incorporación de las estructuras sociales ya comenzaría en una fase anterior a la capacidad de su comprensión en términos racionales por las subjetividad, en la que la sedimentación de los estereotipos sexuales de un macho activo y una hembra pasiva, permiten desde la infancia a los niños de enfatizar su posición jerárquica sobre las niñas. Por lo tanto, el proceso de incorporación es pre-categorial, 
pre-objetivo, pre-conceptual, perceptivo con precisión, pero no pre-culturales, y los seres humanos son los arquitectos de la construcción cultural de la realidad sin ser necesariamente conscientes de esto (Csordas, 1990). Decir que el cuerpo, por lo tanto subjetividad, son una producción cultural, nos permite, de aprender acerca de la construcción de la infancia en la historia y en diferentes culturas como también lo hacemos en nuestro tiempo y en nuestra sociedad, y también de reflexionar y teorizar sobre el hecho que las posiciones de estatus como un niño o un adulto no pueden simplemente ser consideradas como un efecto inevitable y naturalizado del pasar del tiempo, sino que tener este estatus se relaciona con las características culturales y económicas de una época determinada. Si por un lado la infancia nos parece una edad en la que se tiene el propósito de su protección desde los peligros de la sociedad para apoyar su desarollo normal de inteligencia y habilidades, en el otro se hace evidente que la clase dominante tiene el objetivo de forjar una conducta de la infancia para mantener y reproducir el orden social, con una visión del niño como un individuo aún no socializado y como una amenaza potencial.

La infancia, especialmente aquella femenina:

está formada a través de una compresión desproporcionada que ve la interacción de los diferentes conocimientos (medicina, fisonomía, pedagogía, religión, etc.) que a su vez ofrecen toda una serie de técnicas, herramientas, maniobras (vendajes, ejercicios físicos, ropa, alimentos, controles sexuales, etc.). Se aspira a establecer una funcionalidad de los cuerpos, su homeostasis interna, una regularidad (una vez más) normal y productiva (Mariani, 2000:51, nuestra traducción).

El concepto de incorporación, vinculado a la infancia en las prácticas pedagógicas, sirve principalmente para resaltar el poder normalizador y medicalizador, que como veremos en los ejemplos de síndromes como el ADHD e la intersexualidad, trata de neutralizar todas las formas de "agency", o sea acciones que se constituyen como crítica encarnada de las ideologías dominantes. Por tanto, podemos decir con Armstrong (1983) que los niños nerviosos, niños delicados, neuropáticos, inadaptados, difícil, hipersensibles e inestables son todos esencialmente la invención de una nueva forma de ver la infancia. 


\section{El lecho de Procusto de la pedagogía}

En la mitología clásica griega, Procusto, que significa "estirador", es el apodo de un bandido griego llamado Damaste (controlador) o incluso Polipèmone (muchos daños) que, estacionado en el monte Coridallo, en Attica, en el camino sagrado entre Eleusis y Atenas, ofrecía antes posada a los viajeros, pero después los golpeaba con un martillo en una cama excavada en la roca. Las víctimas venían estiradas si eran demasiado cortas, o amputadas si sobresalian de la cama. Esta mitología griega parece encajar con la estandarización y la homologación de la escuela. Los viajeros rasgados por la pedagogía incluyen todos aquellos que no cumplen con los requisitos mínimos del sistema educativo basado en el school failure/success.

Cuando hablamos de estandarización nos referimos al arbitrario proceso para lo cual la trayectoria educativa queda rígidamente estructurada, sin tener en cuenta el ambiente y las subjetividades que la atraviesan. Los programas escolares se dibujan a priori para los estándares pedagógicos, que, incorporado en el plan de estudios, indican los puntos de crecimiento homogéneos. Si la etimología latina de la palabra curriculo se refiere a su sentido de camino y de ruta, esto está lejos de su significado actual. La estandarización también lleva un modelo de aprendizaje lineal, basado en el supuesto de que el acceso a nuevos conocimientos necesita requisitos previos, y en la idea de que la trayectoria curricular tiene que ser organizada en etapas. El aprendizaje se concibe por lo tanto como una mera transmisión de conocimientos, abstraídos del entorno históricosocial y político-económico en el que se producen. Tal método pedagógico, que podemos definir prescriptivo, limita el sentido de la relación que el estudiante puede tener con el material de aprendizaje, provocando un proceso de alienación cultural. Esto también favorece lo que Paulo Freire llama "invasión cultural”, porque el estudiante se vuelve vulnerable a las ideas forzadas (ideas relacionadas con la cultura dominante) y de afuera (ideas propagadas como parte de un proceso de imperialismo cultural). El plan de estudios y, más específicamente, las nuevas pedagogías de la competencia, se basan en la suposición de que las necesidades del desarrollo económico del mercado y las relativas al crecimiento cultural y moral libre de los seres humanos, son perfectamente coincidentes. Términos como "competencia" y "curriculo" se derivan del modelo de la 
“economía del conocimiento", en vez de por las ciencias de la educación (Gleeson y Husbands, 2001). En las escuelas italianas, por ejemplo, en los últimos años para la evaluación que se ha extendido la suministración de las pruebas estandarizadas, cuyo objetivo es examinar la inteligencia utilitaria, funcional dentro del sistema de producción. La suministración de las pruebas se realiza a nivel nacional, por el Istituto Nazionale di Valutazione del Sistema di Istruzione (INVALSI) y, a nivel europeo e internacional, gracias a la Organisation for Economic Co-operation and Development a través del Programme for International Student Assesment (PISA). Desde 2000, el PISA mide las habilidades de los alumnos y a partir de 2012 se comenzó a evaluar también la financial literacy:

un conjunto de conocimiento y capacidad de comprensión de los conceptos financieros combinados con las habilidades, la motivación y la confianza en su propia capacidad, que le permiten aplicar los mismos conocimientos y comprensión para tomar decisiones eficaces en múltiples y diferentes contextos financieros, para mejorar el bienestar de los individuos y de la sociedad y para permitir la participación consciente en la vida económica (INVALSI, 2012, in linea).

Este tipo de pruebas se enmarcan dentro de una nueva estructura de poder después de la crisis de los años 60-70, que ha generado formas de instituciones que intentan perpetuar un nuevo tipo de autoritarismo burocrático, despersonalizando el poder, externalizándolo y poniéndolo en manos del mercado económico. Las pruebas parecen marcar el paso de la "sociedad disciplinaria" de Michel Foucault a la que Gilles Deleuze llama “sociedad de los controles". El concepto más utilizado hoy en día para la legitimidad de esta práctica de evaluación y control es la meritocracia. Esto, por los gobiernos neoliberales es "el marco ideológico en el que el mercado y las desigualdades producidas por el se hacen aceptable e incluso deseable, mientras los sistemas de evaluación estandarizada y cuantitativa introducen al mercado en la escuela, aunque sin nombrarlo" (Borelli, 2013:10, nuestra traducción). La meritocracia permite delegar por completo al individuo los riesgos sociales y económicos, induciendolo a internalizar en forma de culpa personal su condición de exclusión. Ella representa una nueva refundación (reconocimiento y consolidación) de la organización jerárquica de la sociedad y sus organizaciones, haciendo las desigualdades aceptables sobre una base 
esencialmente racional y éticamente legítima. El problema del mérito de hecho no es su desigualdad sino su legitimidad (Hadjar, 2008). Como dice Michael Young (1958), el inventor de esta expresión, la meritocracia refuerza las jerarquías y las élites existentes, garantizando la justeza de sus privilegios, mientras debilita el poder de los que se les oponen.

En Italia el creciente mecanismo de sumisión del sistema educativo al mercado económico, encuentra un punto culminante con la ley sobre la autonomía escolar presentada por el ministro de Berlinguer en 1999. Esta ley, a través del pretexto de permitir la libre determinación de las escuelas, en realidad crea un mecanismo de competencia empresarial. Con la oferta educativa de la escuela, de hecho, se crea la lógica de «préstamos» y «deudas», que representa exactamente lo que Paulo Freire, en su famoso libro Pedagogía del oprimido (1970), llama “educación bancaria”, en la que el acto de enseñar se convierte en aquel de depositar. Esta línea es también la nueva reforma del actual gobierno, La buona scuola (La buena escuela), que se perfila como el cumplimiento de la mercantilización y de la privatización. La crisis en la escuela debido a una economía de mercado neoliberal, por lo tanto, responderá paradójicamente convirtiéndose ella misma en mercado. Podemos hacer una lectura crítica de la crisis en el sistema de educación cada vez más estandarizada analizando "los excluidos" y los "patologizados" de la escuela. Refiriéndose al último informe del Ministero dell'Istruzione per l'Università e la Ricerca (MIUR, 2015) sobre la inclusión escolar, o al informe del Istituto Nazionale di Statistica (ISTAT,2015), vemos que en los últimos años hubo un incremento de los estudiantes con discapacidades en las escuelas públicas, que especifica que en todas las órdenes y los niveles escolares, la discapacidad intelectual (regulada por la ley 104/1992) es la tipología más común, en particular dentro de las escuelas primarias y secundarias, seguido de "otro tipo de discapacidad", una categoría que incluye problemas psiquiátricos tempranos como los Trastornos Específicos del Aprendizaje (regulados por la ley 170/2010), a veces certificados en comorbilidad con otros trastornos, y Síndrome de Déficit de Atención e Hiperactividad (ADHD).

Los sujetos torturados del lecho de Procusto de la school failure, que no cumplen los requisitos mínimos del sistema educativo dictado por el mercado económico, muestran que el aprendizaje es una forma de "consenso político", mientras 
se niega una forma de "resistencia política". En esta dirección, Peter McLaren en Schooling as a Ritual Performance (1986), compara los procesos educativos a un sistema ritual, dividiendo las actividades educativas en dos tipos opuestos entre sí: aquellas que caen en la denominada "estructura de conformidad" y las que son parte de la "anti-estructura de la resistencia". En particular, en este última se incluye toda una serie de actos, también ritualizados, con la que los alumnos oponen resistencia a los mecanismos de control de la escuela, activando un proceso de erosión del poder. Al analizar la estructura de la anti-resistencia, McLaren señala que el comportamiento de oposición de los estudiantes tiene un significado histórico y vivido, que desafía la legitimidad, el poder y el sentido de la educación. Como varios autores han teorizado ampliamente (Rose 2007; Pupavac, 2001; McDermott y Varenne, 1995), la reinterpretación en términos médicos y patológicos de la conducta desviada respecto al orden social dominante, trabaja principalmente en la perspectiva de gestión y control social. Las técnicas de manejo terapéutico del sí (medicalización), lejos de actuar una función de expulsión o castigo de aquellos que se desvían del sistema dominante, constituyen más bien nuevas formas de autoridad política (legitimadas por el saber “científico") que tienen por objeto la normalización del sujeto y su re-inclusión en el mismo sistema. En consecuencia el cuerpo, en el proceso de medicalización, se convierte en una realidad exclusivamente biológica, donde la pedagogía invierte en el poder/saber médico de la prevención y de la cura de la las desviaciones sociales para producir la normalidad: por un lado el poder educativo, con el poder psiquiátrico, establece como se puede identificar lo que son las patologias; por el otro, el poder psiquiátrico hace funcionar la realidad como la escuela (Foucault, 2003). Por tanto, el mecanismo normal de una patologización se convierte en un mecanismo de defensa e instrumento de control social para una pedagogía en crisis.

Para la reflexión que se propone es interesante analizar la (re)acción de patologización de la pedagogía en el caso del "síndrome" de intersexualidad y en el caso del "síndrome" de ADHD, que a través de la psiquiatría intenta normalizar ciertas subjetividades que no están alineadas con los paradigmas culturales preestablecidos. Lo que sucede en la categorización del sexo en Italia es, de hecho, un intento de hacer natural algo construido culturalmente; pero este sistema entra en crisis cuando conoce una condición tal como, por ejemplo, la intersexualidad, donde esta opción no es 
contemplada: una persona no puede permanecer en esta condición, siempre es sólo un estado temporal entendido como enfermedad que tiene que ser curada antes de entrar en una categoria especifica.

En el caso del ADHA, también conocido en Italia como "síndrome de hiperactividad" es interesante observar la perspectiva disciplinaria que se lleva en un intento de normalizar el movimiento considerado "demasiado". Para entender esta enfermedad y su noción "científica" nos basamos en las definiciones oficiales de ADHD que son proporcionadas por los manuales diagnósticos ICD-10 (World Health Organization [WHO], 1992) y DSM-V (American Psychological Association [APA], 2013). Estas definiciones, aunque asumen una causalidad orgánica como causa de la enfermedad, se componen exclusivamente de descripciones del comportamiento observable. Los criterios para la identificación y la evaluación del "niño con ADHD" son, de hecho, construidos en comparación con una expectativa de performance dentro de un contexto institucional específico, que es a menudo el de la escuela. Aunque el DSM-V los define como "síntomas" -como si fueran condición física que resultan de un estado de enfermedad- estas "condiciones" parecen más bien el producto de reglas sociales específicos. Entre todas las enfermedades psiquiátricas infantiles, el "síndrome hipercinético" parece, por tanto, el más adecuado para apoyar esta medicalización del proceso pedagógico, estimulando también en paralelo con una creciente "biologización" de la psiquiatría a través de su estrecha relación con el tratamiento farmacológico: «Among childhood psychiatric disorders, hyperkinetic syndrome held unique promise for a revived biological psychiatry because it was already connected to a specific drug treatment» (Singh, 2008:351). Ya en los años Sesenta, de hecho, el metilfenidato, bajo el nombre comercial Ritalin (producido por la industria farmacéutica Ciba-Geigy, ahora incorporada en el gigante Novartis) - aún el fármaco más utilizado para el tratamiento de ADHD - junto con otros medicamentos estimulantes, fue ampliamente comercializado para la terapia de "diversos problemas de comportamiento" en los niños. En 1989, el metilfenidato se había retirado del mercado italiano y clasificado a la par con otros medicamentos y a partir de julio 2003 a junio 2007 estaba disponible sólo en los centros regionales de referencia; hoy a partir de junio 2007 se puede comprar en la farmacia con la receta de los Centros de referencia regional. Esta comercialización de Ritalin en los últimos años causó un debate entre los “expertos” y en la opinión pública. 
La comunidad psiquiátrica se vio profundamente dividida sobre el valor terapéutico de la administración de psicofármacos a los niños, sobre los efectos secundarios (en particular en el largo plazo) poco conocidos y sobre el conflicto de intereses entre los médicos partidarios de la terapia con medicamentos y los fabricantes de productos farmacéuticos.

La comercialización de sustancias capaces de modificar la conducta humana ha de entenderse como la acción recíproca de las neurociencias y del poder que Rose analizó a través del concepto de «sí neuroquímico». Esa del "sí neuroquímico" es una idea centrípeta del ser humano, donde lo que determina esto último y lo define normal o anormal es sólo dentro de sí mismo, o más bien en el cerebro; todo lo que es externo, el idioma, la cultura, el ambiente, no cuenta. El hombre como "animal político", el "hombre-constelación" y todas las centrífugas definiciones de lo humano se almacenan en la perspectiva neurocientífica. Rose conecta el "nacimiento" de disciplinas Psy al desarrollo de tecnologías de gobierno liberales y afirma que los expertos de estas ciencias han adquirido una posición privilegiada en los siglos pasados, ya que son estas disciplinas que pretenden comprender los determinantes internos de la conducta humana y proporcionar el apoyo adecuado en el conocimiento, en el juicio y en la técnica.

La biopolítica hoy depende por un laboratorio de extensa creación de nuevos fenómenos, por el enorme poder computacional del aparato que busca conectar las historias médicas y las genealogías familiares con secuencias genómicas, por los poderes de mercado de las compañías farmacéuticas, por las estrategias de regulación de la ética de la investigación, por los comités/órganos para la autorización de los medicamentos y los comités de bioética, y por supuesto, por la búsqueda de beneficios y valor de las acciones que esta situación promete. Es aquí, en las prácticas de biopoder contemporáneo que hay que buscar nuevas formas de autoridad (Rose, 2007:40, traducción nuestra).

Por lo tanto las ciencias Psy han jugado un papel constitutivo en las prácticas de sometimiento en las estrategias autoritarias de gobierno de democracias liberales. La politización de esta increíble proliferación técnico-disciplinar científica establecida sobre de existencia, sobre la vida y sobre el cuerpo por el mercado económico, al fin de introducir el término bioeconomía también en las instituciones escolares, ha producido 
sin embargo, en el ejemplo italiano, deferentes críticas de los discursos de la neurociencia. Para rechazar el proceso de normalización ético y político que producen estas ciencias, han nacido nuevas formas de autogobierno y espacios sociales de autoorganización educativa.

\section{La politización del bios}

Como vimos la acción pedagógica, gracias a una estrategia de bio-política que da acceso al cuerpo, es capaz de producir con la disciplina y el control un habitus como un producto de la internalización de los principios de un arbitrario cultural capaz de perpetuarse después cese la acción pedagógica y por tanto perpetuar en las prácticas de los principios del arbitrario internalizado (Bourdieu y Passeron, 1970). Pero existen sujetos no dóciles que practican resistencia al poder y lo fuerzan a recurrir a mecanismos de medicalización y patologización para neutralizar las críticas y las resistencias que se producen en los procesos de escolarización. Así, como para los cuerpos cortados en el lecho de Procusto, la vida se reduce esencialmente a vida desnuda o a la dimensión griega de zoe, es decir, a la simple reducción biológica dada por el discurso neurocientífico que prevé un cuerpo cada vez más atravesado por las técnicas de gobierno. Pero hay una crítica politizada de estos mecanismos, que está apoyando el desarrollo y la difusión de proyectos alternativos de educación (a menudo biosociales) a la escuela tradicional, tanto dentro de las diferentes áreas institucionales que como en los no-institucionales, tales como la comunidad o la familia; en estos lugares se intenta ofrecer el suelo del bios para dar forma y calificar sus vidas, tratando de liberar la subjetividad de la sujeción, para que puedan acceder a producciones creativas por sí mismos. Los cuerpos y la vida en el centro de estos procesos y de la cuestión política, son lugares donde todavía se aplica el poder, el primer estadio de sometimiento, pero al mismo tiempo se convierten en líneas de fuga, en instancias de subjetivaciones, en vehículos creativos y transformativos de acciones socio-políticas. Parafraseando Foucault, la vida, en lo que ocurre en el ejemplo italiano ya mencionado de la red Tutta un'altra scuola, se vuelve en "un objeto político" que es, "en cierto modo, tomada literalmente y volcada contra el sistema que estaba empezando a controlarla" (Foucault, 1994:265, nuestra traducción). 
Particular atención se debe dar por lo tanto a la dimensión del agency que debe entenderse dentro de lo que Michel Foucault llamó "la paradoja de la subjetividad", es decir, el hecho de que la capacidad de acción de un sujeto es posible y se produce dentro de relaciones específicas de poder y subordinación, donde se da al mismo sujeto la oportunidad de practicar el auto-reposicionamiento, contribuyendo a la transformación de las estructuras sociales y políticas. Un principio esto que se refiere a la teoría de los "agentes sociales que determinan de forma activa, a través de categorías de percepción y evaluación socialmente e históricamente determinadas, la situación que los causa" (Bourdieu, 1992: 100-2, nuestra traducción) y que permite relacionarse críticamente frente a los procesos de su construcción/constricción que en este caso está representado por la escuela.

\subsection{El ejemplo italiano de la red nacional Tutta un'altra scuola}

En concreto, toda la red nacional Tutta un'altra scuola, se presenta como "un archipiélago extendido y mellado de experiencias e iniciativas educativas también de largo plazo y para diferentes grupos de edad, en este momento poco conocido y a menudo sumergido pero vivo y deseoso de afirmar su identidad y sus ideas, así como sus prácticas" (Mottana, 2015:8, nuestra traducción). Las prácticas pedagógicas que recoge son muy diferentes entre sí e incluyen las escuelas parentales, libertarias, escuelas en los bosques y toda las escuelas al aire libre, o las múltiples experiencias de homeschooling. Esta red, que se formó en torno a la preocupación común de que "la escuela convencional puede a menudo ser un lugar aplastado bajo la supremacía económica y de mercado, que imponen de contenidos, métodos y procedimientos de selección” (Mottana, 2015:8, nuestra traducción), pone en cambio el objetivo común de “dar respuestas organizadas y conocimientos en un campo surcado por propuestas especímenes, pero también muy diferentes. Además, incentivan a los actores que se mueven en este mundo sumergido a encontrarse y confrontarse, con el único objetivo de establecer nuevas ideas para la formación" y luego alimentar la aspiración de establecer "un modelo de educación y de escuela diferente desde lo que hoy se cristaliza y dentro de lo cual a menudo no se sientan mal sólo los niños, sino también los operadores y las familias" (Mottana, 2015:8, nuestra traducción). 
El camino de la red nacional parece reposicionarse en continuidad con otras rutas críticas en el proceso de escolarización, que especialmente en Italia a principios de los años sesenta/setenta, en un momento de grandes movimientos sociales (antipsiquiátricos, estudiantiles, obreristas, etc.) fueron capaces de poner en cuestión la autoridad de la escuela a nivel político en lugar de didáctico, como lo hizo la pedagogía activista (Bini, 1971). En la famosa obra colectiva L'erba voglio, publicada en 1971, las nuevas formas de autoorganización y la autogestión de las prácticas educativas se consideran como prácticas reales de autogobierno y participación política. Por lo tanto, en las críticas a los procesos de escolarización y en las alternativas educativas como la red Tutta un'altra scuola, se da la oportunidad de volcar en pedagogía la biopolítica como forma política sobre la vida en una biopolítica afirmativa de una política de la vida, donde el fin de la educación nunca es pura supervivencia, sino modus, "formas de vida", hasta llegar a la autogestión de los deseos y a la resolución de problemas éticopolíticos. Por tanto, el escenario que se abre, es el spinozista de una potentia (potencia) contra la potestas (poder), donde el poder está visiblemente encarnado por Procusto, mientras la potencia es de los sujetos desviados que debido a su "excedente" encarnan toda la potencia transformadora que, a pesar del poder disciplinario sobre sus cuerpos, hace que sea posible abrir nuevos lugares de crítica a los procesos educativos. Por lo tanto la escuela no es la separación de la vida, sino la vida misma.

\section{Referencias bibliográficas}

Abberley, P. (1987). The Concept of Oppression and the development of a Social Theory of Disability. En Disability \& Society, 2 (1), pp. 5-19, Routledge.

American Psychological Association, (2013). The Diagnostic and Statistical Manual of Mental Disorders 5. Washington: American Psychiatry Publishing.

Armstrong, D. (1987). Bodies of Knowledge: Foucault and the Problem of Human Anatomy. En G. Scambler, (Ed.) Sociological Theory and Medical Sociology. London: Tavistock.

Barry, A., Osborne, T., Rose, N. (1996). The foucault and political Reason. Liberalism, Neoliberalism and Nationalities of Government. Chicago: Chicago University Press. 
Barton, L. (Ed.) (2001). Disability, Politics and Struggle for Change. London: David Fulton.

Bini, G. (1971). La pedagogia attivistica in Italia. Roma: Editori Riuniti.

Borelli, M. (2013). Le insidie della valutazione. In Gli Asini, n.18, IV, pp. 6-12.

Bourdieu, P. (1992). Risposte. Per una antropologia riflessiva. Bollati Boringhieri: Torino

Bourdieu, P., Passeron, J.C. (1970). La reproduction. Eléments pour une théorie du système d'enseignement. Paris: Les Editions de Minuit.

Burchell, G., Gordon, C., Miller, P. (1991). The Foucault effect: studies in Governmentality. Harvester Wheatsheaf: Hemeln Hempstead.

Busher, H. (2006). Understanding educational leadership: People, power and culture. Buckingham: Open University Press.

Castel, R. (2004). L'insicurezza sociale. Che significa essere protetti? Torino: Enaudi.

Csordas, T. (1990). Embodiment as a Paradigm for Anthropology. En Ethos, vol. 18, pp. 5-47.

Esposito, R. (2004). Bios, biopolitica e filosofia. Torino: Enaudi.

Facchinelli, E., Vaiani, L.M., Sartori, G. (1971). L'erba voglio. Pratica non autoritaria nella scuola, Torino: Enaudi.

Foucault, M. (1975a). Surveiller et punir. Naissance de la prison 1975 Editions Gallimard, Paris.

Foucault, M. (1975b). Pouvoir et corps. En Quel corps?, n. 2, pp. 2-5.

Foucault, M. (1976). Histoire de la sexualité: 1, La volonté de savoir. Paris: Gallimard.

Foucault, M. (1994). Non au sexe roi. En D. Defert, F. Ewald (eds.), Dits it écrtits II 1976-1984 (pp. 256-269). Paris: Gallimard.

Foucault, M. (2003). Le pouvoir psychiatrique: cours au Collège de France, 19731974. Paris: Gallimard.

Freire, P. (1970). Pedagogy of the oppressed. New York: Seabury Press. 
Gleeson, D., Husbands, C. (2001). The Performing School: Managing, Teaching and Learning in a Performance Culture. London: Routledge Falmer.

Hadjar, A. (2008). Meritokratie als Legitimationsprinzip. Die Entwicklung der Akzeptanz sozialer Ungleichheit im Zuge der Bildungsexpansion. Erschienen: Wiesbaden.

$\begin{array}{llllll}\text { INVALSI (2012). } & \text { Consulta } & \text { realizada } & 16 & \text { giugno } & 2017\end{array}$ http://www.invalsi.it/INVALSI/ri/pisa2012.php?page=pisa2012 it_01

ISTAT (2015). Report- L'integrazione degli alunni con disabilità nelle scuole primarie e secondarie di I grado. Consulta realizada 16 giugno 2017 https://www.istat.it/it/files/2015/12/Alunni-condisabilit $\% \mathrm{C} 3 \% \mathrm{~A} 0$. pdf?title=Integrazione + degli + alunni + con + disabilit $\% \mathrm{C} 3 \% \mathrm{~A} 0+$ -+21\%2Fdic $\% 2$ F2015+-+Testo+integrale+e+nota + metodologica.pdf

Lourau R. (1969) L'illusion pedagogique. Paris: EPI

Mariani, A. (2000). Foucault: per una genealogia dell'educazione. Napoli: Liquori Editori.

Massa, R. (1986). Le tecniche e $i$ corpi, verso una scienza dell'educazione. Milano: Edizione Unicopli.

Mcdermott, R., Varenne, H. (1995). Culture as Disability. En Anthropology and Education Quarterly, vol. 26, no. 3, pp. 324-248.

Mclaren, P. (1986). Schooling as a ritual performance. Towards a political economy of educational gestures. London: Routledge \& Kegan Paul.

MIUR, (2015). L'integrazione scolastica degli alunni con disabilità - a.s.2014/2015, Direzione Generale per gli Studi, la Statistica e i Sistemi Informativi, MIUR Ufficio di Statistica. Consulta realizada 16 giugno 2017 http://www.istruzione.it/allegati/2015/L'integrazione_scolastica_degli_alunni_co n_disabilit $\% \mathrm{C} 3 \% \mathrm{~A} 0$ as_2014_2015.pdf

Mottana, P. (2015). Un'altra educazione è possibile. En Tutta Un'Altra Scuola 2015-atti del convegno (pp.6-9). Editrice Aam Terra Nuova.

Oliver, M. (1990). The politics of Disablement. Basingstoke: Macmillan. 
Prout, A. (1989). Sickness as a Dominant Symbol in Life Course Transitions. An illustrated Theoretical Frameworh. En Sociology of Health and Illness, 11 (4), pp. 336-59.

Pupavac, V. (2001). Therapeutic Governance: Psycho-social Intervention and Trauma Risk Management. En Disasters, vol. 2, no. 4, pp. 358-372.

Rose, N. (2007). The Politics of Life Itself. Biomedicine Power, and Subjectivity in the Twenty-First Century. Oxford: Princeton University Press.

Singh, I. (2008). ADHD, culture and education. En Early Child Development and Care. Vol. 178, No. 4, Pp. 347-361.

World Health Organization, (1992). The ICD-10 Classification of Mental and Behavioral Disorders: Clinical Descriptions and Diagnostic Guidelines, 10th revision. Geneva: World Health Organisation.

Young, M. (1958). The rise of the Meritocracy. London: Thames \& Hudson. 